\title{
Scoring rules on dichotomous preferences
}

\author{
Marc Vorsatz
}

Received: 22 June 2006 / Accepted: 17 July 2007 / Published online: 4 September 2007

(C) Springer-Verlag 2007

\begin{abstract}
In this paper, we study individual incentives to report preferences truthfully for the special case when individuals have dichotomous preferences on the set of alternatives and preferences are aggregated in form of scoring rules. In particular, we show that (a) the Borda Count coincides with Approval Voting, (b) the Borda Count is the only strategy-proof scoring rule, and (c) if the size of the electorate is greater than three, then the dichotomous preference domain is the unique maximal rich domain under which the Borda Count is strategy-proof.
\end{abstract}

\section{Introduction}

We analyze the aggregation of preferences in form of positional voting methods or scoring rules (see, Smith 1973; Young 1975, among others) when individuals have dichotomous preferences on the set of alternatives (every alternative belongs either to the set of good or to the set of bad alternatives; an alternative is good if it is weakly preferred to all other alternatives and it is bad if any arbitrary alternative is weakly preferred to it). In particular, we are interested in strategy-proof scoring rules; that is, we look for social choice functions belonging to the class of scoring rules that give

I thank Jordi Massó for his supervision and his never-ending encouragement. Miguel-Ángel Ballester showed me how to improve on earlier drafts of the paper. Salvador Barberà, Carmen Bevía, Bhaskar Dutta, Lars Ehlers, Alejandro Neme, Shmuel Nitzan and Yves Sprumont helped me a lot with their comments. All remaining errors are mine. This research was undertaken with support from the fellowship 2001FI 00451 of the Generalitat de Catalunya and from the research grant BEC2002-02130 of the Ministerio de Ciencia y Tecnología of Spain while I have been a graduate student at Universitat Autònoma de Barcelona.

M. Vorsatz $(\varangle)$

Department of Economics (AE1), Maastricht University, P.O. Box 616, 6200 MD Maastricht, The Netherlands

e-mail:m.vorsatz@algec.unimaas.nl 
individuals incentives to report preferences truthfully. Our main results establish that on this domain restriction, the Borda Count (see, Borda 1781; Young 1974) is equal to Approval Voting (see, Brams and Fishburn 1978) and the only strategy-proof scoring rule.

In a series of papers, Saari and van Newenhizen (1988a, b) and Brams et al. (1988) discuss advantages and disadvantages of Approval Voting versus scoring rules in general and the Borda Count in particular. The former authors argue that Approval Voting is highly indeterminate for a lot of preference profiles (many different outcome can occur given a preference profile) and suggest the Borda Count as an alternative to the widely established Plurality Rule. But this indeterminacy of Approval Voting is rather a virtue according to the latter authors, because it eliminates incentives not to vote sincerely whereas scoring rules are considered to be very manipulable (see, Dummett 1998; Saari 1990; Smith 1999).

One way how to contribute to this discussion is to compare scoring rules with Approval Voting for different preference domains. But this task is not a straightforward one, because the aggregation procedures work quite differently. While scoring rules are social choice functions and thus take into account the whole preference structure, Approval Voting is a voting rule that endows individuals with the right to vote for as many alternatives as they wish to and selects all alternatives with the largest support. Therefore, the level of information available about individual preferences is generally lower under Approval Voting. This problem disappears when preferences are restricted to be dichotomous, because if we interpret voting decisions as the set of good alternatives, then individual preferences are fully revealed; that is, Approval Voting becomes a social choice function. Since this is not true any more for richer preference domains, the dichotomous preference domain constitutes is ideally suited for comparing scoring rules with Approval Voting.

Scoring rules are typically applied on strict preference domains, but if they are generalized to weak preferences in natural way, then the Borda Count is an affine transformation of Approval Voting on the domain of dichotomous preferences (Proposition 1). Since Brams and Fishburn (1978) have shown that Approval Voting is equal to the Condorcet Rule (de Condorcet 1989) on this domain (remember that according to (Inada 1964) the set of Condorcet winners is non-empty on dichotomous preferences), the criticism on the Borda Count not to select an existing Condorcet winner is only true if preferences consist of at least three indifference classes. An intuitive way to appreciate of these conclusions is to recognize that by dividing the space into the two categories of good and bad alternatives, each voter's view of the alternatives loosely resembles what happens when there are only two alternatives. As this classic two-alternative setting is strategy-proof and always has a Condorcet winner, we must anticipate these Approval Voting conclusions.

One common way to eliminate individual incentives to behave strategically is to implement strategy-proof social choice functions. Brams and Fishburn (1978) have shown that Approval Voting is strategy-proof on the dichotomous preference domain and, therefore, one may wonder whether other scoring rules share the same property. Proposition 2 gives a negative answer to this question if there are at least three voters, and therefore, the Borda Count is the best scoring rule in terms of individual incentives given the domain restriction. 
Finally, we deal with the question whether the underlying preference domain can be enlarged without losing strategy-proofness for the Borda Count. Barbie et al. (2006) study strategy-proof domains for the Borda Count under the assumptions that individual preferences are strict and ties are broken in a non-neutral way. They find that the Borda Count is non-manipulable on all domains which contain one fixed preference relation and all its cyclic permutation. Since these domains are rather small, their result confirms the common opinion that scoring rules are highly manipulable. Proposition 3 points into the same direction, because the dichotomous preference domain is the largest domain for which the Borda Count is strategy-proof whenever the size of the electorate is larger than three.

We proceed as follows. Next, we introduce notation and some basic definitions. Afterwards, we present our results. In a final section, we justify the way we define scoring rules for weak preferences by means of an axiomatic analysis.

\section{Notation and definitions}

Consider a group of individuals $N=\{1, \ldots, n\}$ with preferences on the set of alternatives $K$. The cardinalities of the two sets are finite and equal to $n \geq 2$ and $k \geq 3$. We assume that $k \geq 3$, because otherwise all scoring rules are equal to the Borda Count as it will become clear from the definitions later on. Elements of $K$ are usually denoted by $x, y$ and $z$.

Let $R_{i}$ be the weak preference relation of individual $i$ on $K$. We assume that $R_{i}$ is reflexive, complete, and transitive. The strict and the indifference preference relations associated with $R_{i}$ are denoted by $P_{i}$ and $I_{i}$, respectively. The set of all weak preference relations on $K$ is denoted by $\mathcal{R}$. A domain $\overline{\mathcal{R}}$ is a subset of $\mathcal{R}$. Given a domain $\overline{\mathcal{R}} \subseteq \mathcal{R}$, a preference profile $R=\left(R_{1}, \ldots, R_{n}\right) \in \overline{\mathcal{R}}^{N}$ is an $n$-tuple of individual preference relations. The $i$-variant preference profile $\left(R_{i}^{\prime}, R_{-i}\right)$ is obtained by changing the preference relation of individual $i$ at profile $R$ from $R_{i}$ to $R_{i}^{\prime} \in \overline{\mathcal{R}}$.

Given the preference relation $R_{i} \in \overline{\mathcal{R}}$, an alternative is good (bad) for individual $i$ if it is weakly preferred to any other alternative (if any arbitrary alternative is weakly preferred to it). Formally, define the set of good alternatives associated with $R_{i}$ as $G\left(R_{i}\right)=\left\{x \in K: x R_{i} y\right.$ for all $\left.y \in K\right\}$. Similarly, let $B\left(R_{i}\right)=\{x \in K$ : $y R_{i} x$ for all $\left.y \in K\right\}$ be the set of bad alternatives corresponding to $R_{i}$. The cardinalities of these sets are equal to $g\left(R_{i}\right)$ and $b\left(R_{i}\right)$. The preference relation $R_{i} \in \overline{\mathcal{R}}$ is then said to be dichotomous if and only if every alternative belongs to at least one of these two sets: that is, if and only if $G\left(R_{i}\right) \cup B\left(R_{i}\right)=K$. The domain of all dichotomous preferences is denoted by $\mathcal{D} \subset \mathcal{R}$ and $D_{i} \in \mathcal{D}$ is a particular dichotomous preference relation for individual $i$. Finally, given the dichotomous preference profile $D=\left(D_{1}, \ldots, D_{n}\right) \in \mathcal{D}^{N}$, let $I(D)=\left\{i \in N: G\left(D_{i}\right)=K\right\}$ be the set of unconcerned individuals and $N_{x}(D)=\left|\left\{i \notin I(D): x \in G\left(D_{i}\right)\right\}\right|$ be the concerned support for $x$ at $D$.

A social choice function $f: \overline{\mathcal{R}}^{N} \rightarrow 2^{K} \backslash\{\emptyset\}$ selects for all preference profiles $R \in \overline{\mathcal{R}}^{N}$ a non-empty set of alternatives $f(R)$. Any social choice function belonging to the class of scoring rules can be represented by a vector $s=\left(s_{0}, s_{1}, \ldots, s_{k-1}\right) \in \mathbb{R}^{k}$ satisfying the conditions $s_{j-1} \leq s_{j}$ for all $j=1, \ldots, k-1$ and $s_{0}<s_{k-1}$. The range of 
$s$ is normalized by assuming that $s_{0}=0$ and $s_{k-1}=k-1$. Scoring rules are typically applied on the domain of strict preferences $\mathcal{P}$. In this case, points are assigned to every alternative in such a way that if alternative $x$ is in the $j$ 'th top position according to $P_{i}$, then $x$ receives $p_{x}^{s}\left(P_{i}\right)=s_{k-j}$ points from individual $i$. Given a strict preference profile $P \in \mathcal{P}^{N}$ and an alternative $x \in K$, let $p_{x}^{s}(P)=\sum_{i=1}^{n} p_{x}^{s}\left(P_{i}\right)$ be the score of alternative $x$ at $P$ when scoring rule $s$ is applied. Then, society selects for a given strict preference profile the set of alternatives with the highest score.

However, if preferences are not strict, then the point assignment process has to be generalized. If we aim to study extensions which are neutral (if an individual is indifferent between two alternatives, both alternative receive the same amount of points from that individual), maintain the total amount of points constant for every preference relation, and assign to an alternative the same amount of points whenever its relative position with respect to two preference relation is identical, then we have to do it as follows (see Sect. 4 for a formal proof): Given the preference relation $R_{i} \in \overline{\mathcal{R}}$ that consists of $c\left(R_{i}\right)$ indifference classes, $1 \leq c\left(R_{i}\right) \leq k$, let $C^{m}\left(R_{i}\right)$ be the set of alternatives belonging to the $m$ 'th top indifference class of $R_{i}$. The cardinality of $C^{m}\left(R_{i}\right)$ is $c^{m}\left(R_{i}\right)$. Moreover, denote by $Q^{m}\left(R_{i}\right)$ the union of the top $m$ indifference classes of $R_{i}$; that is, $Q^{m}\left(R_{i}\right)=\bigcup_{j=1}^{m} C^{j}\left(R_{i}\right)$. The corresponding cardinality is $q^{m}\left(R_{i}\right)$. Then, for all $m, 1 \leq m \leq c\left(R_{i}\right)$, all alternatives $x \in C^{m}\left(R_{i}\right)$ receive $p_{x}^{s}\left(R_{i}\right)=\frac{1}{c^{m}\left(R_{i}\right)} \sum_{j=1}^{c^{m}\left(R_{i}\right)} s_{\left(k-q^{m-1}\left(R_{i}\right)-j\right)}$ points from individual $i$ when scoring rule $s$ is applied. This approach is the obvious and natural one of using the average of the relevant scores. Given the preference profile $R \in \overline{\mathcal{R}}^{N}$ and alternative $x \in K$, let $p_{x}^{S}(R)=\sum_{i=1}^{n} p_{x}^{S}\left(R_{i}\right)$ be the score of alternative $x$ at $R$ under $s$.

Definition 1 The social choice function $f_{s}: \overline{\mathcal{R}}^{N} \rightarrow 2^{K} \backslash\{\emptyset\}$ associated with the scoring rule $s$ is such that for all $R \in \overline{\mathcal{R}}^{N}, x \in f(R)$ if and only if $p_{x}^{s}(R) \geq p_{y}^{s}(R)$ for all $y \in K$.

The Borda Count $f_{B}$ is given by $s_{j}=j$ for all $j=0, \ldots, k-1$. With a slight abuse of notation by dropping the superscript $s$ we write $p_{x}\left(R_{i}\right)$ and $p_{x}(R)$ whenever the Borda Count is applied. Finally, we provide some intuition for the generalized point assignment process corresponding to the Borda Count: Given $R_{i} \in \overline{\mathcal{R}}$, compare alternative $x$ with every alternative $y \in K \backslash\{x\}$. If $x P_{i} y$, then assign one point to $x$ and zero points to $y$ (give the point to $y$ whenever $y P_{i} x$ ). If $x I_{i} y$, then split the point equally. The sum of points alternative $x$ obtains after performing all pair-wise comparisons is equal to $p_{x}\left(R_{i}\right)$.

Approval Voting is one of the most prominent voting rules both in theory and practice. It endows individuals with the right to vote for not just one but for as many alternatives as they wish to and, given the votes from all individuals, the set of all alternatives with the highest number of votes is selected: that is, the mapping $M_{i}: \overline{\mathcal{R}} \rightarrow 2^{K}$ determines for all preference relations $R_{i} \in \overline{\mathcal{R}}$ the set of alternatives $M_{i}\left(R_{i}\right) \in 2^{K}$ individual $i$ votes for and the Approval Voting function $v:\left(2^{K}\right)^{N} \rightarrow 2^{K} \backslash\{\emptyset\}$ aggregates the individual voting decisions by selecting the alternatives with the highest number of votes. Hence, for all $\left(M_{1}\left(R_{1}\right), \ldots, M_{n}\left(R_{n}\right)\right) \in\left(2^{K}\right)^{N}, x \in v\left(M_{1}\left(R_{1}\right), \ldots, M_{n}\left(R_{n}\right)\right)$ if and only if $\left|\left\{i \in N: x \in M_{i}\left(R_{i}\right)\right\}\right| \geq\left|\left\{i \in N: y \in M_{i}\left(R_{i}\right)\right\}\right|$ for all $y \in K$. 
Regenwetter and Tsetlin (2004) discuss different probabilistic models that make assumptions on how the mappings $\left(M_{i}\right)_{i \in N}$ look like in order to compare Approval Voting in expected terms to social choice functions. But for the case of dichotomous preferences the natural interpretation of the mappings $M_{i}: \mathcal{D} \rightarrow 2^{K}$ is such that for all $i \in N$ and all $D_{i} \in \mathcal{D}, M_{i}\left(D_{i}\right)=G\left(D_{i}\right) .{ }^{1}$ Thus, we are able to define Approval Voting as a social choice function.

Definition 2 The social choice function $f: \mathcal{D}^{N} \rightarrow 2^{K} \backslash\{\emptyset\}$ is Approval Voting if for all $D \in \mathcal{D}^{N}, x \in f(D)$ if and only if $N_{x}(D) \geq N_{y}(D)$ for all $y \in K$.

The social choice function corresponding to Approval Voting is denoted by $f_{A}$. A transition of Approval Voting to social choice functions does not exist any more if preferences are richer, because, given $M_{i}\left(R_{i}\right)$ for a particular preference relation $R_{i} \in \overline{\mathcal{R}}$ that consists of at least three indifference classes, it is impossible to recover preferences fully. To see this let the preference relation $P_{i} \in \mathcal{P}$ be such that $x P_{i} y P_{i} z$. In this case, we cannot infer from $M_{i}\left(P_{i}\right)=\{x, y\}$ that $x P_{i} y$. Similarly, if $M_{i}\left(P_{i}\right)=\{x\}$, then we cannot deduce that $y P_{i} z$.

\section{Results}

The dichotomous preference domain is well suited for comparing Approval Voting and scoring rules, because it is the largest domain for which Approval Voting constitutes a well-defined social choice function (in the sense that it is possible to recover preferences fully from votes). Claim 1 states that, given a preference relation, the difference in points between a good and a bad alternative is constant whenever the Borda Count is applied. In particular, the difference does not depend on the size of the set of good alternatives.

Claim 1 If $D_{i} \in \mathcal{D}$ satisfies $x P_{i} y$, then $p_{x}\left(D_{i}\right)-p_{y}\left(D_{i}\right)=\frac{k}{2}$.

Proof Let the scoring rule $s$ be equal to the Borda Count and consider the preference relation $D_{i} \in \mathcal{D}$ which is such that $x P_{i} y$. From the equation $\sum_{j=1}^{n} j=\frac{n(n+1)}{2}$ we deduce that alternative $x$ receives $p_{x}\left(D_{i}\right)=\frac{\sum_{j=1}^{g\left(D_{i}\right)} k-j}{g\left(D_{i}\right)}=\frac{g\left(D_{i}\right) k-\sum_{j=1}^{g\left(D_{i}\right)} j}{g\left(D_{i}\right)}=$ $\frac{2 g\left(D_{i}\right) k-g\left(D_{i}\right)\left(g\left(D_{i}\right)+1\right)}{2 g\left(D_{i}\right)}=\frac{2 k-g\left(D_{i}\right)-1}{2}$ points from individual $i$. Observe that $k-g\left(D_{i}\right) \stackrel{2}{=} b\left(D_{i}\right)$, because $D_{i} \in \mathcal{D}$ belongs to a concerned individual. Therefore, $i$ assigns $p_{y}\left(D_{i}\right)=\frac{\sum_{j=1}^{b\left(D_{i}\right)} k-g\left(D_{i}\right)-j}{b\left(D_{i}\right)}=\frac{b\left(D_{i}\right)\left(k-g\left(D_{i}\right)\right)-\sum_{j=1}^{b\left(D_{i}\right)} j}{b\left(D_{i}\right)}=$ $\frac{2 b\left(D_{i}\right)\left(k-g\left(D_{i}\right)\right)-b\left(D_{i}\right)\left(b\left(D_{i}\right)+1\right)}{2 b\left(D_{i}\right)}=\frac{k-g\left(D_{i}\right)-1}{2}$ points to alternative $y$. We conclude that $p_{x}\left(D_{i}\right)-p_{y}\left(D_{i}\right)=\frac{k}{2}$.

It follows immediately that the score of an alternative according to the Borda Count is an affine transformation of the number of concerned individuals who approve it.

\footnotetext{
1 An individual that votes for all alternatives or none at all reveals to be unconcerned. Since the effect of both strategies is the same under Approval Voting, there is no loss of generality by setting $M_{i}\left(D_{i}\right)=K$. The alternative would be to let $M_{i}\left(D_{i}\right)$ be equal to $K \backslash B\left(D_{i}\right)$.
} 
Said differently, the Borda Count is equivalent to Approval Voting on the dichotomous preference domain.

Proposition 1 For all $D \in \mathcal{D}^{N}, f_{B}(D)=f_{A}(D)$.

Proof Let the scoring $s$ be equal to the Borda Count. Given the preference profile $D \in$ $\mathcal{D}^{N}$ and alternative $x \in K, p_{x}(D)=\frac{k}{2} N_{x}(D)+\sum_{i \notin I(D)} \frac{k-g\left(D_{i}\right)-1}{2}+\frac{k-1}{2} \cdot|I(D)|$ by Claim 1. We conclude that the difference in the score of $x$ and $y$ is equal to $p_{x}(D)-p_{y}(D)=\frac{k}{2}\left(N_{x}(D)-N_{y}(D)\right)$. It follows that for all $D \in \mathcal{D}^{N}, N_{x}(D) \geq$ $N_{y}(D)$ for all $y \in K$ if and only if $p_{x}(D) \geq p_{y}(D)$ for all $y \in K$.

One aim of the literature on social choice theory is to study normative properties of aggregation procedures. Special attention should be given to strategy-proof social choice functions, because they assure that individuals have incentives to represent their preferences truthfully, or, to say it differently, all room for individual strategic behavior is eliminated. So far, we cannot define strategy-proofness properly, because the image of a social choice function is set-valued but preferences are defined on alternatives. One way how to deal with this problem is to extend preferences. In particular, we assume that the reflexive, complete, and transitive preference relation $\succsim_{R_{i}}$ defined on $2^{K} \backslash\{\emptyset\}$ satisfies the subsequent properties proposed by Brams and Fishburn (1978):

1. Condition P: $\{x\} \succ_{R_{i}}\{x, y\} \succ_{R_{i}}\{y\}$ if and only if $x P_{i} y$.

2. Condition R: For all $S, T \subseteq 2^{K} \backslash\{\emptyset\}$, if $S \subseteq G\left(R_{i}\right)$ or $T \subseteq B\left(R_{i}\right)$ or $[S \backslash T \subseteq$ $G\left(R_{i}\right)$ and $\left.T \backslash S \subseteq B\left(R_{i}\right)\right]$, then $S \succsim R_{i} T .^{2}$

One preference extension that satisfies condition $\mathrm{P}$ and $\mathrm{R}$ for dichotomous preferences is expected utility maximization (an individual cares about the proportion of good alternatives selected), the case for which Bogomolnaia et al. (2005) obtain a series of impossibility results. Now, we can define strategy-proofness in a straightforward way. The social choice function $f: \overline{\mathcal{R}}^{N} \rightarrow 2^{K} \backslash\{\emptyset\}$ is manipulable by $i$ on $\overline{\mathcal{R}}^{N}$ if for some $R \in \overline{\mathcal{R}}^{N}$ and $R_{i}^{\prime} \in \overline{\mathcal{R}}, f\left(R_{i}^{\prime}, R_{-i}\right) \succ_{R_{i}} f(R)$.

Definition 3 The social choice function $f: \overline{\mathcal{R}}^{N} \rightarrow 2^{K} \backslash\{\emptyset\}$ is strategy-proof on $\overline{\mathcal{R}}$ if it is not manipulable by any individual on $\overline{\mathcal{R}}^{N}$.

Approval Voting is strategy-proof on the dichotomous preference domain due to Brams and Fishburn (1978). The proof that any scoring rule different from the Borda Count is manipulable on this domain whenever at least three individuals participate in the election is based on the observation that for other scoring rules, the difference in points between a good and a bad alternative depends on the size of the set of good alternatives.

\footnotetext{
${ }^{2}$ Condition $\mathrm{R}$ implies that adding a good (bad) alternative to a set of alternatives containing only good (bad) alternatives leaves individuals indifferent, whereas individuals would be strictly better (worse) off according to the separable preferences studied by Barberà et al. (1991). This difference stems from the fact that the latter authors emphasize situations where alternatives are compatible, here we interpret the image of a social choice function as the set of pre-selected alternatives from which a unique alternative will be selected afterwards.
} 
Claim 2 Suppose $D_{i}^{t} \in \mathcal{D}$ is such that $x P_{i} y$ and $g\left(D_{i}^{t}\right)=t, 0<t<k$. Only the Borda Count satisfies $p_{x}^{s}\left(D_{i}^{t}\right)-p_{y}^{s}\left(D_{i}^{t}\right)=c$ for all $t$.

Proof Let the preference relation $D_{i}^{t} \in \mathcal{D}$ be such that $x P_{i} y$ and $g\left(D_{i}^{t}\right)=t, 0<t<k$, and suppose that there exists a scoring rule $s$ different from the Borda Count for which $p_{x}^{s}\left(D_{i}^{t}\right)-p_{y}^{s}\left(D_{i}^{t}\right)=c$ for all $t$. Let $T$ be the total amount of points assigned by the scoring rule $s$. Considering $t=1$, we have that $p_{x}^{s}\left(D_{i}^{1}\right)-p_{y}^{s}\left(D_{i}^{1}\right)=k-1-\frac{T-(k-1)}{k-1}=$ $k-\frac{T}{k-1}$. Similarly, if $t=k-1$, then $p_{x}^{s}\left(D_{i}^{k-1}\right)-p_{y}^{s}\left(D_{i}^{k-1}\right)=\frac{T}{k-1}-0$. Since both numbers are equal to $c$ by assumption, $k-\frac{T}{k-1}=\frac{T}{k-1}$ or $T=\frac{k(k-1)}{2}$. Since $t$ can take $k-1$ different values, we conclude that $c=\frac{k}{2}$.

Finally, we prove by induction that $c=\frac{k}{2}$ implies necessarily $s_{j}=j$ for all $j=1, \ldots, k-2$. We know that $p_{x}\left(D_{i}^{k-1-1}\right)-p_{y}\left(D_{i}^{k-1-1}\right)=\frac{T-s_{1}}{k-2}-\frac{s_{1}}{2}=\frac{k}{2}$. This equation is equivalent to $s_{1}=1$. Let $q \geq 2$ and suppose that for all $\bar{q}<q$, $p_{x}\left(D_{i}^{k-1-\bar{q}}\right)-p_{y}\left(D_{i}^{k-1-\bar{q}}\right)=\frac{T-\sum_{j=1}^{\bar{q}} s_{j}}{k-\bar{q}-1}-\frac{\sum_{j=1}^{\bar{q}} s_{j}}{\bar{q}+1} \stackrel{!}{=} \frac{k}{2}$ implies $s_{\bar{q}}=\bar{q}$. We have to show that this statement is also true for $q$. Observe that the difference in points, $p_{x}\left(D_{1}^{k-1-q}\right)-p_{y}\left(D_{1}^{k-1-q}\right)$, is equal to $\frac{T-\frac{q(q-1)}{2}-s_{q}}{k-1-q}-\frac{\frac{q(q-1)}{2}+s_{q}}{q+1}$ due to the induction hypothesis. Since this difference has to be equal to $\frac{k}{2}$ and $T=\frac{k(k-1)}{2}$, we yield $\left(\frac{k(k-1)}{2}\right)(q+1)-k \cdot \frac{q(q-1)}{2}-k \cdot s_{q}=\frac{k}{2}(k-1-q)(q+1)$. The result $s_{q}=q$ follows from simple algebra.

Proposition 2 Suppose that $n \geq 3$. The social choice function $f_{s}: \mathcal{D}^{N} \rightarrow 2^{K} \backslash\{\emptyset\}$ corresponding to the scoring rule s is strategy-proof if and only if it is the Borda Count.

Proof The Borda Count is strategy-proof on the dichotomous preference domain according to Brams and Fishburn (1978) and Proposition 1. To show that any scoring rule $s$ different from the Borda Count is manipulable on this preference domain, note that by Claim 2 there are two preference relations $D_{i}^{1}, D_{i}^{t} \in \mathcal{D}$, satisfying $G\left(D_{i}^{1}\right)=\{x\}, x \in G\left(D_{i}^{t}\right), y \notin G\left(D_{i}^{t}\right)$ and $g\left(D_{i}^{t}\right)=t, 1<t<k$, such that $p_{x}^{s}\left(D_{i}^{1}\right)-p_{y}^{s}\left(D_{i}^{1}\right) \neq p_{x}^{s}\left(D_{i}^{t}\right)-p_{y}^{s}\left(D_{i}^{t}\right)$.

Let $K=\left\{x, y, z_{1}, \ldots, z_{k-2}\right\}$ and consider the following profile $\left(D_{i}^{1}, D_{-i}\right) \in \mathcal{D}^{N}$ : $G\left(D_{i}^{1}\right)=\{x\}, G\left(D_{j}\right)=\{y\}$ and $G\left(D_{l}\right)=\{x, y\}$ for all $l \neq i, j$. In this case, $f_{s}\left(D_{i}^{1}, D_{-i}\right)=\{x, y\}$ because $n \geq 3$. Let $D_{i}^{t} \in \mathcal{D}$ correspond to set of good alternatives $G\left(D_{i}^{t}\right)=\left\{x, z_{1}, \ldots, z_{t-1}\right\}$. If $p_{x}^{s}\left(D_{i}^{1}\right)-p_{y}^{s}\left(D_{i}^{1}\right)>p_{x}^{s}\left(D_{i}^{t}\right)-p_{y}^{s}\left(D_{i}^{t}\right)$, then $f_{s}\left(D_{i}^{t}, D_{-i}\right)=\{y\}$ and individual $i$ can manipulate $f_{s}$ at $\left(D_{i}^{t}, D_{-i}\right) \in \mathcal{D}^{N}$ via $D_{i}^{1}$. If $p_{x}^{s}\left(D_{i}^{1}\right)-p_{y}^{s}\left(D_{i}^{1}\right)<p_{x}^{s}\left(D_{i}^{t}\right)-p_{y}^{s}\left(D_{i}^{t}\right)$, then $f_{s}\left(D_{i}^{t}, D_{-i}\right)=\{x\}$ and individual $i$ can manipulate $f_{s}$ at $\left(D_{i}^{1}, D_{-i}\right) \in \mathcal{D}^{N}$ via $D_{i}^{t}$.

The results are mixed for $n=2$. To see this suppose that $K=\{x, y, z\}$ and let the preference profile $\left(D_{1}, D_{2}\right)$ be such that $G\left(D_{1}\right)=\{x, z\}$ and $G\left(D_{2}\right)=\{y\}$. For this preference profile we find that $f_{s}(D)=\{y\}$ whenever $s_{1}<1$. If $D_{1}^{\prime}$ is the preference relation corresponding to $G\left(D_{1}^{\prime}\right)=\{x\}$, then $f_{s}\left(D_{1}^{\prime}, D_{2}\right)=\{x, y\}$. Since individual 1 with the preference relation $D_{1}$ strictly prefers $\{x, y\}$ to $\{y\}$ according to condition $\mathrm{P}$, we have found a viable manipulation whenever $s_{1}<1$. On the other hand, one 
can show by means of straightforward calculus that any scoring rule corresponding to the values $s_{1} \geq 1$ is strategy-proof on the dichotomous preference domain for some reflexive, complete, and transitive preference extension satisfying condition $\mathrm{P}$ and condition R. One example is the situation when individuals are expected utility maximizers.

Next, we analyze whether the domain restriction can be weakened. That is, are there domains containing the set of dichotomous preferences under which the Borda Count is strategy-proof? Following Ching and Serizawa (1998), the domain $\overline{\mathcal{R}} \subseteq \mathcal{R}$ is a maximal domain for a list of properties for the social choice function $f: \overline{\mathcal{R}}^{N} \rightarrow 2^{K} \backslash\{\emptyset\}$ if (a) $f: \overline{\mathcal{R}}^{N} \rightarrow 2^{K} \backslash\{\emptyset\}$ satisfies the list of properties, and (b) for all $\tilde{\mathcal{R}} \supsetneq \overline{\mathcal{R}}$, $f: \tilde{\mathcal{R}}^{N} \rightarrow 2^{K} \backslash\{\emptyset\}$ does not satisfy the list of properties. In addition to strategyproofness we consider a richness condition that eliminates all small domains for which the Borda Count is strategy-proof. The condition we apply is stronger than the one of Berga and Serizawa (2000) who propose that the domain $\overline{\mathcal{R}} \subseteq \mathcal{R}$ is rich if for all $x \in K$ there exists a preference relation $R_{i} \in \overline{\mathcal{R}}$ such that $x P_{i} y$ for all $y \in K \backslash\{x\}$. Here, the domain $\overline{\mathcal{R}} \subseteq \mathcal{R}$ is rich if for all $x \in K$ there exists a dichotomous preference relation $D_{i} \in \overline{\mathcal{R}}$ such that $G\left(D_{i}\right)=\{x\}$.

Claim 3 If the preference relation $R_{i} \in \overline{\mathcal{R}}$ is such that $c\left(R_{i}\right) \geq 3, x \in G\left(R_{i}\right)$ and $y \in B\left(R_{i}\right)$, then $p_{x}\left(R_{i}\right)-p_{y}\left(R_{i}\right)>\frac{k}{2}$.

Proof Let the preference relation $R_{i} \in \overline{\mathcal{R}}$ be such that $c\left(R_{i}\right) \geq 3, x \in G\left(R_{i}\right)$ and $y \in B\left(R_{i}\right)$. We have that $p_{x}\left(R_{i}\right)-p_{y}\left(R_{i}\right)=\frac{1}{g\left(R_{i}\right)} \sum_{j=1}^{g\left(R_{i}\right)}(k-j)-\frac{1}{b\left(R_{i}\right)} \sum_{j=1}^{b\left(R_{i}\right)-1} j$ is strictly greater than $\frac{1}{g\left(R_{i}\right)} \sum_{j=1}^{g\left(R_{i}\right)}(k-j)-\frac{1}{b\left(R_{i}\right)} \sum_{j=1}^{k-g\left(R_{i}\right)-1} j$ because $c\left(R_{i}\right) \geq 3 \mathrm{im}$ plies $k-g\left(R_{i}\right)>b\left(R_{i}\right)$. Consider the dichotomous preference relation $D_{i}^{\prime} \in \mathcal{D}$ which is such that $x P_{i}^{\prime} y$ and $g\left(D_{i}^{\prime}\right)=g\left(R_{i}\right)$. Then, $p_{x}\left(D_{i}^{\prime}\right)-p_{y}\left(D_{i}^{\prime}\right)=\frac{1}{g\left(R_{i}\right)} \sum_{j=1}^{g\left(R_{i}\right)}(k-$ $j)-\frac{1}{b\left(R_{i}\right)} \sum_{j=1}^{k-g\left(R_{i}\right)-1} j$. Since this difference is equal to $\frac{k}{2}$ by Claim 1 , we conclude that $p_{x}\left(R_{i}\right)-p_{y}\left(R_{i}\right)>\frac{k}{2}$.

Proposition 3 The dichotomous preference domain is a maximal domain for strategy-proofness for the Borda Count whenever $n \geq 3$. If $n>3$, then it is the unique maximal one.

Proof It is obvious that the dichotomous preference domain is rich. Moreover, the Borda Count is strategy-proof on $\mathcal{D}$ according to Brams and Fishburn (1978) and Proposition 1. To construct a viable manipulation, consider the preference relation $R_{i} \in \overline{\mathcal{R}}$ which is such that $c\left(R_{i}\right) \geq 3, x \in G\left(R_{i}\right)$ and $y \in B\left(R_{i}\right)$.

If $n$ is even, then let the preference profile $D \in \mathcal{D}^{N}$ be such that $G\left(D_{i}\right)=\{x\}$ for all $i \leq \frac{n}{2}$ and $G\left(D_{i}\right)=\{y\}$ for all $i>\frac{n}{2}$. If $n$ is odd, then the preference profile $D \in \mathcal{D}^{N}$ satisfies $G\left(D_{i}\right)=\{x\}$ for all $i \leq \frac{n-1}{2}, G\left(D_{i}\right)=\{y\}$ for all $\frac{n-1}{2}<i<n-1$ and $G\left(D_{n}\right)=\{z\}$ for some $z \neq x, y$. We have that $f_{B}(D)=\{x, y\}$ if $n>3$. Moreover, $f_{B}\left(R_{1}, D_{-1}\right)=\{x\}$ by Claim 1 and 3 . Thus, individual 1 can manipulate the Borda Count at $D \in \mathcal{D}^{N}$ via $R_{i}$ if $n>3$. Since the preferences used to construct the manipulation belong to any rich domain different from $\mathcal{D}$, we have shown maximality and uniqueness simultaneously whenever $n>3$. Uniqueness can not be proved for 
the case $n=3$, but we are able to obtain maximality by considering the preference profile $D \in \mathcal{D}^{N}$ which is such that $G\left(D_{1}\right)=\{x\}, G\left(D_{2}\right)=\{y\}$ and $G\left(D_{3}\right)=\{x, y\}$. In this case, individual 1 can manipulate the Borda Count at $D \in \mathcal{D}^{N}$ via $R_{i}$, because $f_{B}(D)=\{x, y\}$ and $f_{B}\left(R_{1}, D_{-1}\right)=\{x\}$ by Claim 1 and 3 .

Consider the following example to see why there is another maximal rich domain for strategy-proofness for the Borda Count if the number of individuals is equal to three.

Example 1 Suppose that $n=3$ and $K=\{x, y, z\}$. Let the preference domain $\overline{\mathcal{R}}=\left\{D_{i}, D_{j}, D_{l}, R_{i}\right\}$ be completely prescribed by the sets $G\left(D_{i}\right)=\{x\}, G\left(D_{j}\right)=$ $\{y\}, G\left(D_{l}\right)=\{z\}, G\left(R_{i}\right)=\{x\}$, and $B\left(R_{i}\right)=\{z\}$. Note that the domain $\overline{\mathcal{R}}$ is rich. If the preference profile $R \in \overline{\mathcal{R}}$ is such that two individuals have the same preference relation $D_{m}, m=i, j, l$, or one individual has the preference relation $D_{i}$ and a second individual has the preference relation $R_{i}$, then the Borda Count selects the top alternative according to $D_{m}$ or alternative $x$, respectively. We can see that at these preference profiles the top alternative of two individuals is chosen. Since the third individual cannot change this by misrepresenting her/his preferences, there are only two possible manipulations: Individual $i$ either manipulates the Borda Count at $\left(D_{i}, D_{j}, D_{l}\right) \in \overline{\mathcal{R}}^{N}$ via $R_{i} \in \overline{\mathcal{R}}$ or she/he manipulates the Borda Count at $\left(R_{i}, D_{j}, D_{l}\right) \in \overline{\mathcal{R}}^{N}$ via $D_{i} \in \overline{\mathcal{R}}$. Observe that $f_{B}\left(D_{i}, D_{j}, D_{l}\right)=K$ and $f_{B}\left(R_{i}, D_{j}, D_{l}\right)=\{y\}$. If individual $i$ with the preference relation $D_{i}$, or $R_{i}$ respectively, is indifferent between $\{y\}$ and $\{x, y, z\}$ (this does not contradict neither condition $\mathrm{P}$ nor condition $\mathrm{R}$ ), then the Borda Count is strategy-proof on the $\left\{D_{i}, D_{j}, D_{l}, R_{i}\right\}$ domain.

Finally, Propositions 2 and 3 together imply the following Theorem.

Theorem 1 The dichotomous preference domain is a maximal rich domain where strategy-proof scoring-rules exist.

\section{An axiomatic generalization of scoring rules}

To our best knowledge, the only existing definition of scoring rules that is applicable on weak preference domains is due Myerson (1996). Following his approach the social choice function $f$ is a scoring rule if there exists a set of functions $\left\{S_{x}: \overline{\mathcal{R}} \rightarrow\right.$ $\mathbb{R}\}_{x \in K}$, one for every alternative, such that for all $x \in K, x \in f(R)$ if and only if $x \in \underset{y}{\operatorname{argmax}} \sum_{R_{i} \in \overline{\mathcal{R}}} S_{y}\left(R_{i}\right) \cdot N\left(R_{i} ; R\right)$, where $N\left(R_{i} ; R\right)$ denotes the number of indi-

viduals with preference relation $R_{i}$ given profile $R{ }^{3}$ Since the definition of scoring rules we propose is very intuitive but more restrictive, we would like to support it by means of an axiomatic analysis. To this end, we do not aim at characterizing our class of scoring rules from the set of all social choice functions, rather we want to know how a given scoring rule for strict preferences should be extended to weak preference

\footnotetext{
3 In fact, Myerson's definition of a social choice function is slightly more complicated than the one used in this paper, because it also accounts for a variable set of individuals.
} 
domains. ${ }^{4}$ Formally, let $\tilde{p}_{x}^{s}\left(R_{i}\right)$ be the amount of points individual $i$ with preference relation $R_{i} \in \overline{\mathcal{R}}$ assigns to alternative $x$ if $s$ is applied on $\mathcal{P}$. We want $\left(\tilde{p}_{x}^{s}\left(R_{i}\right)\right)_{x \in K}$ to satisfy the following properties.

1. Neutrality If $R_{i} \in \overline{\mathcal{R}}$ satisfies $x I_{i} y$ for some $x, y \in K, \tilde{p}_{x}^{s}\left(R_{i}\right)=\tilde{p}_{y}^{s}\left(R_{i}\right)$.

2. Total Points For all $R_{i} \in \overline{\mathcal{R}}, \sum_{x \in K} \tilde{p}_{x}^{s}\left(R_{i}\right)=\sum_{j=1}^{k-1} s_{j}$.

3. Independence For all $R_{i}, R_{i}^{\prime} \in \overline{\mathcal{R}}$ which are such that for some $x \in K,\{y \in$ $\left.K: x R_{i} y\right\}=\left\{y \in K: x R_{i}^{\prime} y\right\}$ and $\left\{y \in K: y R_{i} x\right\}=\left\{y \in K: y R_{i}^{\prime} x\right\}$, $\tilde{p}_{x}^{s}\left(R_{i}\right)=\tilde{p}_{x}^{s}\left(R_{i}^{\prime}\right)$.

Usually, scoring rules are considered to be neutral in the sense that if the set of alternatives is permuted for a given preference profile, then set of elected alternatives has to be permuted accordingly. Here, neutrality means that no individual discriminates between two alternatives whenever she/he is indifferent between them. Total points states that any individual distributes the same total amount of points irrespective of her/his preferences. And finally, according to independence an alternative which is in the same relative position with respect to two preference relations gets the same amount of points.

Proposition 4 Suppose that the scoring rule $s$ is applied on $\mathcal{P}$. Given $R_{i} \in \overline{\mathcal{R}}$, $\left(\tilde{p}_{x}^{s}\left(R_{i}\right)\right)_{x \in K}$ satisfies neutrality, total points, and independence if and only if for all indifference classes $C^{m}\left(R_{i}\right), 1 \leq m \leq c\left(R_{i}\right)$, and all alternatives $y \in C^{m}\left(R_{i}\right)$, $\tilde{p}_{y}^{s}\left(R_{i}\right)=p_{y}^{s}\left(R_{i}\right)$.

Proof Given the scoring rule $s$ for strict preference domains and the preference relation $R_{i} \in \overline{\mathcal{R}}$, it is easy to see that $p_{x}^{s}\left(R_{i}\right)$ satisfies the three properties. Suppose now that $\tilde{p}_{x}^{s}\left(R_{i}\right)$ satisfies neutrality, total points, and independence. We prove that for all indifference classes $C^{m}\left(R_{i}\right), 1 \leq m \leq c\left(R_{i}\right)$, and all alternatives $x \in C^{m}\left(R_{i}\right)$, $\tilde{p}_{x}^{s}\left(R_{i}\right)=p_{x}^{s}\left(R_{i}\right)$.

Consider any arbitrary indifference class $C^{m}\left(R_{i}\right)$. Take any preference relation $R_{i}^{\prime} \in \overline{\mathcal{R}}$ such that for all $x, y \in C^{m}\left(R_{i}\right)$ and all $w, z \notin C^{m}\left(R_{i}\right), x I_{i}^{\prime} y$, either $w P_{i}^{\prime} z$ or $z P_{i}^{\prime} w$, and $x R_{i}^{\prime} z$ if and only $x R_{i} z$ (this preference relation breaks all possibly existing ties outside $C^{m}\left(R_{i}\right)$ maintaining the relative ordering for all alternatives in $C^{m}\left(R_{i}\right)$ the same). By independence,

$$
\tilde{p}_{x}^{s}\left(R_{i}^{\prime}\right)=\tilde{p}_{x}^{s}\left(R_{i}\right) \text { for all } x \in C^{m}\left(R_{i}\right)
$$

Take any strict preference relation $P_{i}^{\prime \prime} \in \mathcal{P}$ which is such that for all $x, y \in K$, if $x P_{i}^{\prime} y$ then $x P_{i}^{\prime \prime} y$ (this preference relation breaks all ties within $C^{m}\left(R_{i}^{\prime}\right)$ in an arbitrary

\footnotetext{
4 For weak preference domains Myerson's (1996) main result can be stated as follows: a social choice function (with a variable set of individuals) satisfies reinforcement, overwhelming majority, and neutrality if and only if it is a scoring rule (in his sense). Reinforcement asks that if two disjoint groups of individuals elect some common alternatives, then exactly this set has to be elected whenever all individuals within the two groups and no individual outside the two groups participates in the election. Overwhelming majority requires that for any two preference profiles, there exists a natural number $n$ such that at an election consisting of at least $n$ times the first preference profile plus the second preference profile, a subset of the image at the first preference profile is elected. Finally, neutrality states that if the set of alternatives is permuted for a given preference profile, then set of elected alternatives has to be permuted accordingly. This result is clearly unrelated to our characterization.
} 
way). By construction of $P_{i}^{\prime \prime} \in \mathcal{P}$ and the assumption that the scoring rule $s$ is applied for strict preferences, $\sum_{x \notin C^{m}\left(R_{i}\right)} \tilde{p}_{x}^{s}\left(P_{i}^{\prime \prime}\right)=\sum_{j=1}^{k-1} s_{k-j}-\sum_{j=1}^{c^{m}\left(R_{i}\right)} s_{\left(k-q^{m-1}\left(R_{i}\right)-j\right)}$. Since, additionally, for all $x \notin C^{m}\left(R_{i}\right), \tilde{p}_{x}^{s}\left(R_{i}^{\prime}\right)=\tilde{p}_{x}^{s}\left(P_{i}^{\prime \prime}\right)$ by independence and $\sum_{x \in K} \tilde{p}_{x}^{s}\left(R_{i}^{\prime}\right)=\sum_{x \in K} \tilde{p}_{x}^{s}\left(P_{i}^{\prime \prime}\right)=\sum_{j=1}^{k-1} s_{k-j}$ by total points, we conclude that $\sum_{x \in C^{m}\left(R_{i}\right)} \tilde{p}_{x}^{s}\left(R_{i}^{\prime}\right)=\sum_{j=1}^{c^{m}\left(R_{i}\right)} s_{\left(k-q^{m-1}\left(R_{i}\right)-j\right)}$. Hence, by Eq. (1),

$$
\sum_{x \in C^{m}\left(R_{i}\right)} \tilde{p}_{x}^{s}\left(R_{i}\right)=\sum_{j=1}^{c^{m}\left(R_{i}\right)} s_{\left(k-q^{m-1}\left(R_{i}\right)-j\right)} .
$$

The result follows from Eq. (2) and neutrality.

This characterization is tight as the following three examples show.

Neutrality Take alternatives $x$ and $y$ as given. Now, let $\left(\tilde{p}_{z}^{s}\left(R_{i}\right)\right)_{z \in K}$ be such that for all preference relations $R_{i} \in \overline{\mathcal{R}}$ which satisfy $x I_{i} y$ and $x P_{i} z$ for all $z \neq y$, $\tilde{p}_{x}^{s}\left(R_{i}\right)=s_{k-1}+s_{k-2}, \tilde{p}_{y}^{s}\left(R_{i}\right)=0$, and $\tilde{p}_{z}^{s}\left(R_{i}\right)=p_{z}^{s}\left(R_{i}\right)$ for all $z \notin\{x, y\}$. For all other preference relations $R_{i}^{\prime} \in \overline{\mathcal{R}}, \tilde{p}_{z}^{s}\left(R_{i}^{\prime}\right)=p_{z}^{s}\left(R_{i}^{\prime}\right)$ for all $z \in K$. Observe that $\left(p_{z}^{s}\left(R_{i}\right)\right)_{z \in K}$ satisfies total points and independence. The following example shows that it does not satisfy neutrality. Let $K=\{w, x, y\}$ and consider the preference relation $R_{i} \in \overline{\mathcal{R}}$ such that $x I_{i} y P_{i} w$. Since $\tilde{p}_{x}^{s}\left(R_{i}\right)=s_{k-1}+s_{k-2}$ and $\tilde{p}_{y}^{s}\left(R_{i}\right)=0$, $\left(\tilde{p}_{z}^{s}\left(R_{i}\right)\right)_{z \in K}$ does not satisfy neutrality.

Total points Take alternatives $x$ and $y$ as given. Now, let $\left(\tilde{p}_{z}^{s}\left(R_{i}\right)\right)_{z \in K}$ be such that for all preference relations $R_{i} \in \overline{\mathcal{R}}$ which satisfy $x I_{i} y$ and $x P_{i} z$ for all $z \neq y$, $\tilde{p}_{x}^{s}\left(R_{i}\right)=\tilde{p}_{y}^{s}\left(R_{i}\right)=s_{k-1}+1$ and $\tilde{p}_{z}^{s}\left(R_{i}\right)=p_{z}^{s}\left(R_{i}\right)$ for all $z \notin\{x, y\}$. For all other preference relations $R_{i}^{\prime} \in \overline{\mathcal{R}}, \tilde{p}_{z}^{s}\left(R_{i}^{\prime}\right)=p_{z}^{s}\left(R_{i}^{\prime}\right)$ for all $z \in K$. Observe that $\left(p_{z}^{s}\left(R_{i}\right)\right)_{z \in K}$ satisfies neutrality and independence. The following example shows that it does not satisfy total points. Let $K=\{w, x, y\}$ and consider the preference relation $R_{i} \in \overline{\mathcal{R}}$ such that $x I_{i} y P_{i} w$. Since $\sum_{z \in K} \tilde{p}_{z}^{s}\left(R_{i}\right)=2 s_{k-1}+2>s_{k-1}+s_{k-2}$, $\left(\tilde{p}_{z}^{s}\left(R_{i}\right)\right)_{z \in K}$ does not satisfy total points.

Independence Take alternative $x$ as given. Now, let $\left(\tilde{p}_{z}^{s}\left(R_{i}\right)\right)_{z \in K}$ be such that for all preference relations $R_{i} \in \overline{\mathcal{R}}$ which satisfy $x P_{i} y$ for all $y \neq x$ and $y I_{i} z$ for some $y, z \neq x, \tilde{p}_{x}^{s}\left(R_{i}\right)=s_{k-1}+1, \tilde{p}_{y}^{s}\left(R_{i}\right)=p_{y}^{s}\left(R_{i}\right)-\frac{1}{c^{2}\left(R_{i}\right)}$ for all $y \in C^{2}\left(R_{i}\right)$, and $\tilde{p}_{z}^{s}\left(R_{i}\right)=p_{z}^{s}\left(R_{i}\right)$ for all $z \notin\left\{C^{2}\left(R_{i}\right) \cup\{x\}\right\}$. For all other preference relations $R_{i}^{\prime} \in \overline{\mathcal{R}}, \tilde{p}_{z}^{s}\left(R_{i}^{\prime}\right)=p_{z}^{s}\left(R_{i}^{\prime}\right)$ for all $z \in K$. Observe that $\left(p_{z}^{s}\left(R_{i}\right)\right)_{z \in K}$ satisfies neutrality and total points. The following example shows that it does not satisfy independence. Let $K=\{w, x, y\}$ and consider the preference relation $R_{i} \in \overline{\mathcal{R}}$ such that $x P_{i} y I_{i} w$. Then, $\tilde{p}_{x}^{s}\left(R_{i}\right)=s_{k-1}+1$. On the other hand, if $R_{i}^{\prime} \in \overline{\mathcal{R}}$ satisfies $x P_{i}^{\prime} y P_{i}^{\prime} w$, then $\tilde{p}_{x}^{s}\left(R_{i}\right)=s_{k-1}$. Hence, $\left(\tilde{p}_{z}^{s}\left(R_{i}\right)\right)_{z \in K}$ does not satisfy independence. 


\section{References}

Barberà S, Sonnenschein H, Zhou L (1991) Voting by committees. Econometrica 59:595-609

Barbie M, Puppe C, Tasnádi A (2006) Non-manipulable domains for the Borda count. Econ Theory 27:411430

Berga D, Serizawa S (2000) Maximal domain for strategy-proof rules with one public good. J Econ Theory 90:39-61

Bogomolnaia A, Moulin H, Stong R (2005) Collective choice under dichotomous preferences. J Econ Theory 122:165-184

Borda J (1781) Mémoire sur les élections au Scrutin. Histoire de l'Academie Royale des Sciences, Paris

Brams S, Fishburn P (1978) Approval voting. Am Polit Sci Rev 72:831-847

Brams S, Fishburn P, Merrill III S (1988) The responsiveness of approval voting: comments on Saari and van Newenhizen. Publ Choice 59:121-131

Ching S, Serizawa S (1998) A maximal domain for the existence of strategy-proof rules. J Econ Theory 78:157-166

de Condorcet M (1989) An essay on the application of probability decision making: an election between three candidates (1785). In: Sommerlad F, Mc Lean I (eds) The political theory of Condorcet. University of Oxford, Oxford

Dummett M (1998) The Borda count and agenda manipulation. Soc Choice Welf 15:289-296

Inada K (1964) A note on the simple majority decision rule. Econometrica 32(4):525-531

Myerson R (1996) Axiomatic derivation of scoring rules without the ordering assumption. Soc Choice Welf 12:59-74

Regenwetter M, Tsetlin I (2004) Approval voting and positional voting methods: inference, relationship, examples. Soc Choice Welf 22:539-566

Saari D (1990) Susceptibility to manipulation. Publ Choice 61:21-41

Saari D, van Newenhizen J (1988a) Is approval voting an 'Unmitigated Evil'?: a response to Brams, Fishburn, and Merill. Publ Choice 59:132-147

Saari D, van Newenhizen J (1988b) The problem of indeterminacy in approval, multiple, and truncated voting systems. Publ Choice 59:101-120

Smith D (1999) Manipulability measures of common social choice functions. Soc Choice Welf 16:639-661

Smith J (1973) Aggregation of preferences with variable electorates. Econometrica 6:1027-1041

Young P (1974) An axiomatization of Borda's rule. J Econ Theory 9:43-52

Young P (1975) Social choice scoring functions. SIAM J Appl Math 28:824-838 